\title{
DETERMINATION OF LUNG SOUND AS NORMAL OR ABNORMAL, USING A STATISTICAL TECHNIQUE
}

\author{
Isuri Liyanage ${ }^{1}$, Githendri Siriwardhana ${ }^{1}$, Anjana Abeyrathne ${ }^{1}$, Asela Pallewela ${ }^{2}$, \\ Kanchana Wijesinghe ${ }^{1}$ \\ ${ }^{l}$ General Sir John Kotelawala Defence University, Ratmalana, Sri Lanka \\ ${ }^{2}$ Sri Jayawardhanapura General Hospital, Sri Lanka
}

\begin{abstract}
In this study the authors investigate a possibility of objectively differentiating a lung sound as normal or abnormal using a statistical technique. For the study, breath sounds were recorded from 30 nonsmoking, healthy subjects and 7 subjects with respiratory disorders whose external physical symptoms were not shown, using an electronic stethoscope. A 4th order Butterworth bandpass filter removed environment sounds and an Adaptive filter using Least Mean Square algorithm cancelled other body sounds from the recorded sound to obtain only the lung sound. After amplifying a lung sound signal up to the initial recorded amplitude, signal was compared with a standard normal and a standard abnormal lung sound. The comparison was done by calculating the Mahalanobis Distance mean values. The Mahalanobis distance mean values obtained from subjects with respiratory disorders showed considerable deviations from the specific range of values obtained by subjects with normal lung sounds concluding this method is capable of distinguishing between normal and abnormal lung sounds and could developed to noninvasively determine the progress of patients with respiratory disorders.
\end{abstract}

Keywords: Adaptive Noise Cancellation, Least Mean Square Algorithm, Lung Sound Analysis, Mahanaobis Distance

\section{Introduction}

Normal lung sounds are generated either by rapid fluctuations of gas pressure or oscillations of solid tissues while abnormal lung sounds occur due to deformation or obstruction of respiratory track. Normal lung sounds can be categorized to tracheal, bronchial, broncho-vesicular and vesicular (Kandaswamy et al, 2004). They are concentrated in the frequency range of $50 \mathrm{~Hz}-250 \mathrm{~Hz}$ (Sovijarvi et al, 2000, Vannuccini et al, 2000). Abnormal lung sounds can also be categorized to continuous and discontinuous. Continuous abnormal lung sounds can be further classified as wheeze, rhonchus and stridor while discontinuous abnormal lung sound as crackles (Bouzakine et al, 2005). Abnormal lung sounds are concentrated in the range of $150 \mathrm{~Hz}-2500 \mathrm{~Hz}$ (Sovijarvi et al, 2000). The normal and abnormal lung sounds are categorized based on pitch, intensity, location and inspiratory to expiratory duration.

Normal and abnormal lung sounds are analysed to determine pulmonary disorders. Wheeze sound indicates that the person is susceptible to asthma or pneumonia and rhonchus sound indicates chronic obstructive pulmonary disorder or bronchitis while crackles indicate pneumonia, pulmonary fibrosis or congestive heart failure (Chowdhury and Majumder, 1982). Auscultation is the traditional method of determining pulmonary disorders while other noninvasive methods such as pulmonary function test, respiratory inductance plethysmograph, and phonopneumography techniques are also used. But invasive methods such as computerized tomography (CT) scan, chest X ray and bronchoscopy are also used to diagnose the pulmonary disorders. The existing noninvasive diagnostic procedures are mostly subjective while invasive diagnostic procedures are expensive, time consuming and some are harmful. Therefore with the development of automation, many researchers are conducting researches to automatically detect pulmonary disorders non-invasively. For that as the initial stage, 
researchers have investigated computerized methods to classify a lung sound as normal or abnormal and have developed methods to identify some types of lung sounds such as wheezes, crackles and rhonchus automatically.

The analyses of lung sounds by computerized methods have been done using statistical and machine learning techniques. Statistical methods such as higher order crossing discrimination analysis, analysis of variance and machine learning techniques such as artificial neural network (Guler et al, 2005), hidden Markov model (Matsunaga et al, 2009), fuzzy analysis (Zolnoori et al, 2012), autoregressive model (Mendez et al, 2008) and mel frequency cepstrum coefficients (Chang et al, 2010) were used in lung sound analysis.

In this paper, the aim is to explore whether the normal and abnormal lung sound patterns could be compared using Mahalanobis distance statistical method and thereby to determine whether a person's lung sound is normal. This is also a validation of the use of Mahalanobis distance to analyse lung sounds.

\section{Theoretical Background of Mahalanobis distance}

The Mahalanobis distance is one of the most common measures in multivariate statistics. It can be used to determine whether a sample is an outlier or whether a sample has a similarity with another group or not (McLachlan, 1999). The mathematical definition of Mahalanobis distance is given by equation (1).

$$
\mathrm{D}(\mathrm{X}, \mathrm{Y})=\sqrt{ }(\mathrm{X}-\mathrm{Y})^{\mathrm{T}} * \mathrm{~S}^{-1} *(\mathrm{X}-\mathrm{Y})
$$

Where $\mathrm{D}$ is the Mahalanobis distance matrix of data points $(\mathrm{X})$ of the sample matrix to the data points in parent matrix. $\mathrm{Y}$ is the mean of the parent matrix. $\mathrm{S}^{\wedge}(-1)$ is the inverse of covariance matrix of parent matrix and $\mathrm{T}$ is the transpose (Mahalanobis, 1936). In Mahalanobis distance comparison, if two similar matrixes are compared, the mean value of Mahalanobis distance is equals to 1.

\section{Methodology}

\section{Data Acquisition}

This study was performed on 30 ( 15 male and 15 female) nonsmoking healthy subjects who lived a minimum of 4 years in Rathmalana area in Sri Lanka with no known lung, heart or renal diseases and 7 subjects with respiratory disorders in the same area, falling to the age group 19-35 whose having a Body Mass Index (BMI) in the range of 18.5-24.9. Having taken the consent of the participants to the study, each was given a questionnaire to be filled. Then, one person at a time was taken into a room with less interference of background noise but, not sound proofed where, he/she was asked to sit on a stool and relax for 5 minutes. The breath sound at the posterior lower region of the right lung was directly recorded on to the MATLAB software by the electronic stethoscope model "Spirit CK-E600". The subject was asked to hold the breath for the first 10 seconds of the recording and then to take deep breaths in next 50 seconds. The characteristics of 7 subjects with respiratory disorders are as shown in Table 1. 
Isuri Liyanage, Githendri Siriwardhana, Anjana Abeyrathne, Asela Pallewela, Kanchana Wijesinghe / Determination Of Lung Sound As Normal Or Abnormal, Using A Statistical Technique

Table 1 The characteristics of 7 subjects with respiratory disorders

\begin{tabular}{cccc}
\hline Index & Description & Male & Description \\
\hline A & $\begin{array}{c}\text { Has had wheezing condition within one year } \\
\text { before the recording of breath sound, no } \\
\text { external physical symptoms shown. }\end{array}$ & $\mathrm{D}$ & $\begin{array}{c}\text { Has had wheezing condition within one } \\
\text { year before the recording of breath sound, } \\
\text { no external physical symptoms shown. }\end{array}$ \\
\hline B & $\begin{array}{c}\text { Has had wheezing condition within the one } \\
\text { year before the recording of breath sound, no } \\
\text { external physical symptoms shown. }\end{array}$ & $\mathrm{E}$ & $\begin{array}{c}\text { Has had wheezing condition within one } \\
\text { year before the recording of breath sound, } \\
\text { no external physical symptoms shown. }\end{array}$ \\
\hline $\mathrm{C}$ & $\begin{array}{c}\text { Has had wheezing condition within one year } \\
\text { before the recording of breath sound, no } \\
\text { external physical symptoms shown. }\end{array}$ & $\mathrm{F}$ & $\begin{array}{c}\text { Had detected low lung volume by a } \\
\text { pulmonary function test within one year } \\
\text { before the recording of breath sound. }\end{array}$ \\
\hline & $\begin{array}{c}\text { Had detected low lung volume by a } \\
\text { pulmonary function test within two years } \\
\text { before the recording of breath sound. }\end{array}$ \\
\hline
\end{tabular}

The acquired breath sounds which are seen as in Figure 1 were arranged in 16 bit Mono audio format with sampling frequency of $44100 \mathrm{~Hz}$ and stored in .wav file format.

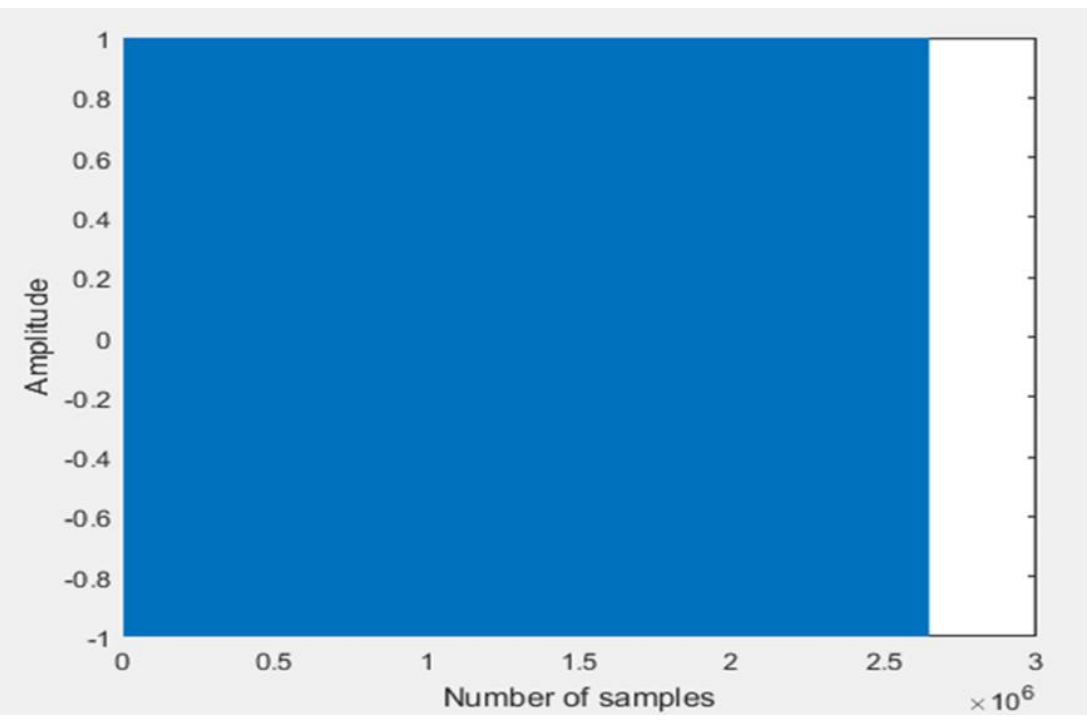

Figure 1: Plotted breath sound after capturing through electronic stethoscope on to MATLAB. The signal is not seen due to external background noise

\section{Background noise filtering}

Since there was a high amount of noise in the recorded signal, a Butterworth bandpass $4^{\text {th }}$ order filter with a cutoff frequency at $100 \mathrm{~Hz}$ and $400 \mathrm{~Hz}$ was designed on MATLAB. Then, the recorded signal is filtered to remove environment noise as seen in Figure 2. Each sound file recorded from the electronic stethoscope was filtered. 


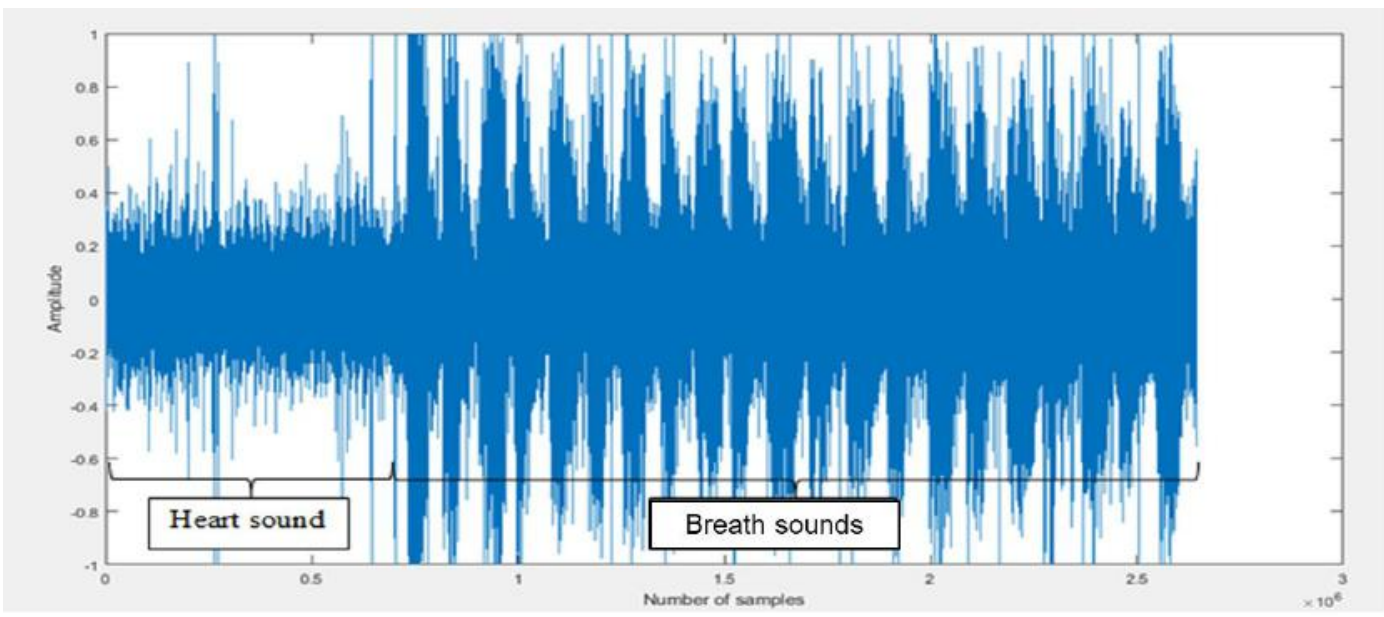

Figure 2: Environment noise filtered waveform of the recorded sound, clearly indicating the heart and breath sounds components. It is seen that the heart and other body sounds have overlapped with lung sounds in the breath sound regions

\section{Extraction of lung sounds}

It can be seen in Figure 2 that mainly heart sounds interfere with lung sounds because the heart sound frequencies which is in the range of $20 \mathrm{H}-150 \mathrm{~Hz}$ overlaps with lung sound frequencies (Hadjileontiadis and Panas, 1997). Therefore a Least Mean Square Adaptive filter was used to acquire only the lung sound (Sathesh and Muniraj, 2012). First the recorded portion of the heart sound of the filtered recording where, the subject was asked to hold the breath (the heart sound) was selected manually, while listening to the audio files and observing the wave patterns of both acquired and standard heart and lung sounds. Then, it was aligned with a selected breath cycle of the above filtered recording where the subject was asked to breath heavily (the breath sound). After that, these two signals were fed into the least mean square adaptive filter designed on MATLAB with a step size of 0.0001 and filter length of 1 where, the output obtained was observed to be a cycle containing only the lung sound as seen in Figure 4. Similarly, 3 consecutive inspiration- expiration cycles of each filtered breath sound were run through the Least Mean Square adaptive filter. The Figure 3 demonstrates the operation of the Adaptive filter (Potdar et al, 2015).

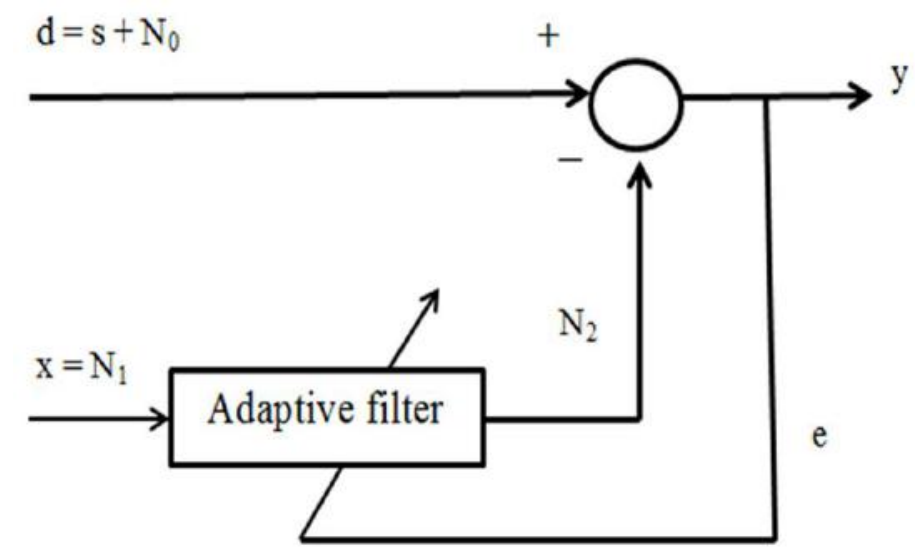

Figure 3: Diagram of Least Mean Square adaptive filter where $d=$ breath sound, $s=$ lung sound, $\mathrm{N}_{0}=$ heart sound and noise, $x=$ heart sound and noise, $\mathrm{N}_{2}=$ adaptive filtered output, $\mathrm{y}=$ lung sound and $\mathrm{e}=$ error 


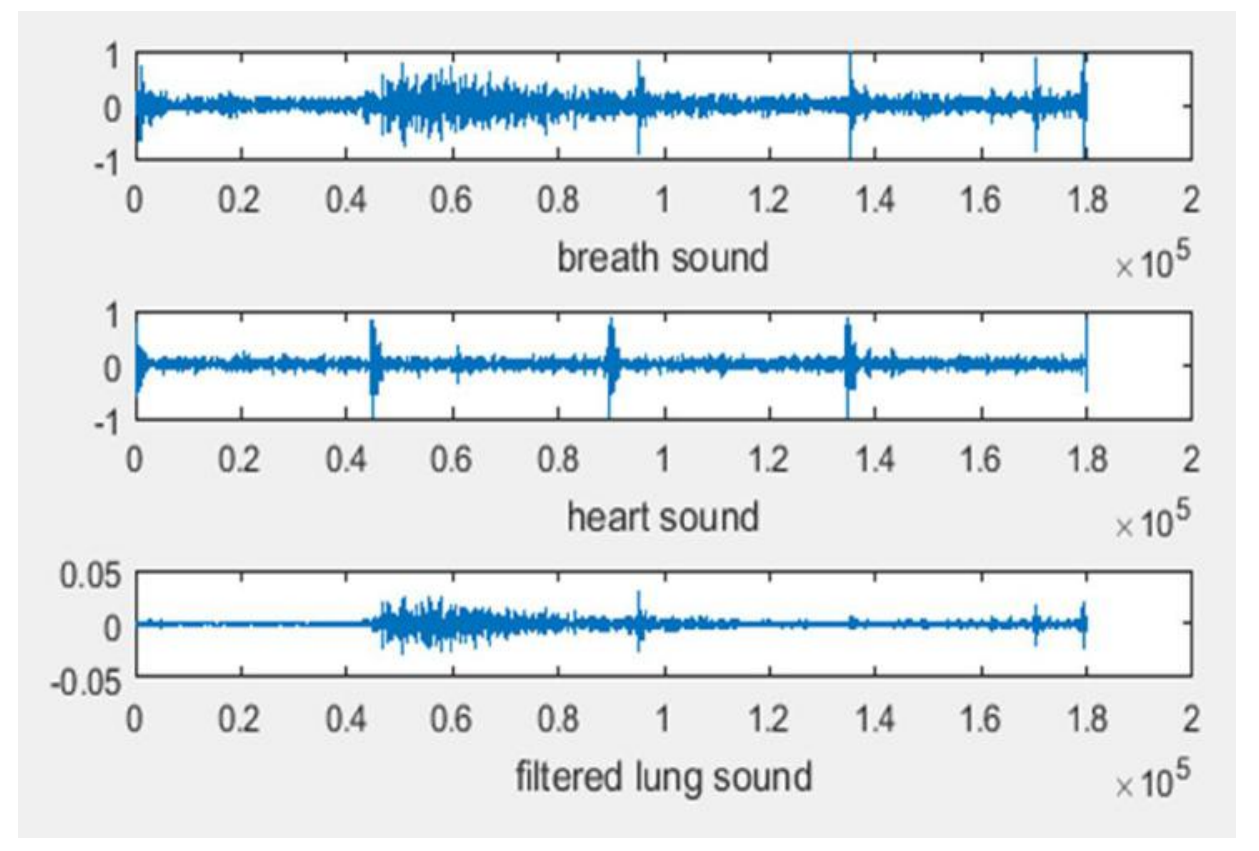

Figure 4: The external noise filtered breath sound, the heart sound and the adaptive filtered lung sound waveforms

Next the power of the adaptive filtered lung sound is restored to the power of the filtered breath sound to eliminate the power losses of the lung sound signal due to adaptive filtering.

\section{Comparison}

The recorded normal lung sounds were compared with the standard normal lung sound using Mahalanobis distance method and obtained a mean value for each normal lung sound and plotted all the mean values in a graph. Then the normal lung sounds were compared with standard abnormal lung sound and plotted the mean values. The standard lung sounds were collected from the lung sound library in R.A.L.E. ${ }^{\circledR}$ Repository. The Figure 5 and Figure 6 shows the standard normal lung sound and standard abnormal lung sound respectively. Then a range was formed using the plotted values where $90 \%$ of the values are to be in the range. Next the recorded abnormal lung sounds were compared with standard normal lung sound and also with standard abnormal lung sound and plotted all the mean values in two separate graphs as previously. Then it was observed whether the values obtained for the abnormal lung sounds are in the formed range or whether they have deviated from the range. 


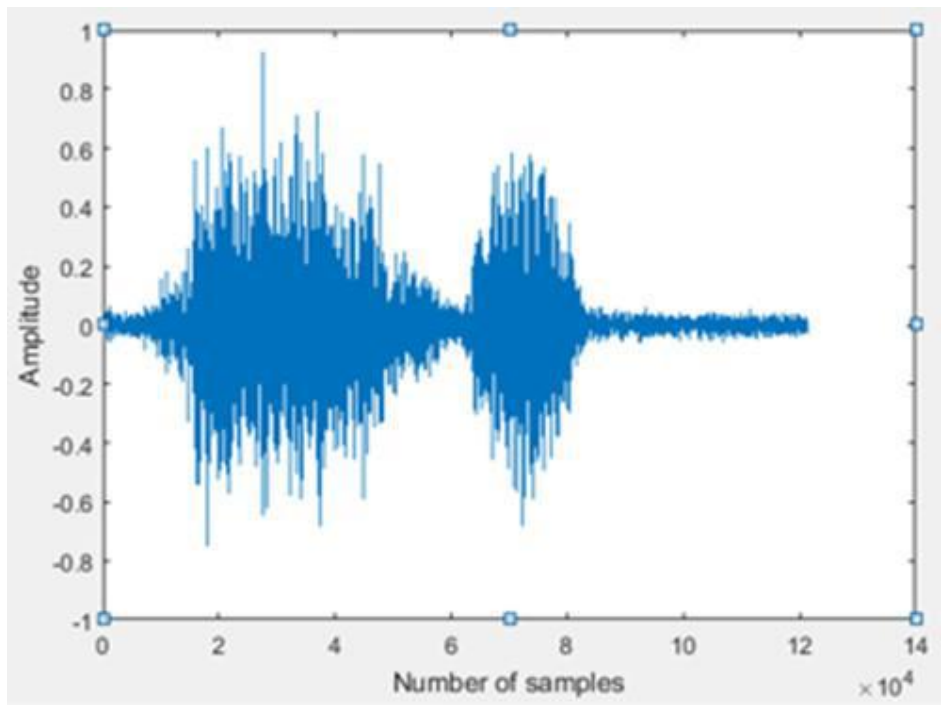

Figure 5: Standard normal lung sound which shows inspiration and expiration

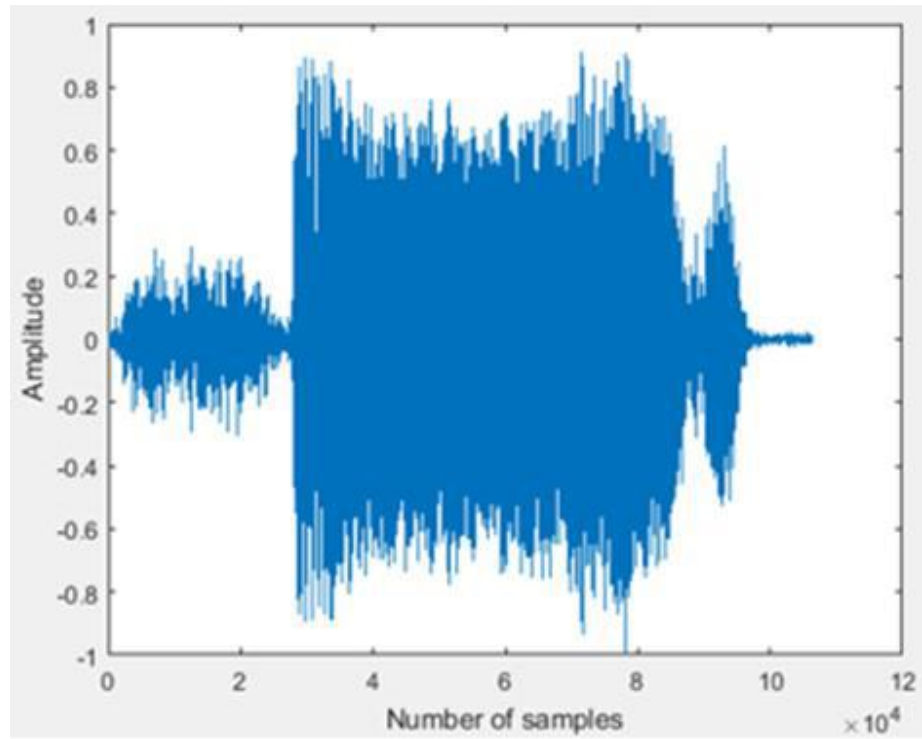

Figure 6: Standard abnormal lung sound which is a wheeze sound

\section{Results and Discussion}

The Mahalanobis distance mean values of each normal lung sound when compared with standard normal lung sound are as shown in Figure 7 and when compared with standard abnormal lung sound are as shown in Figure 8. 

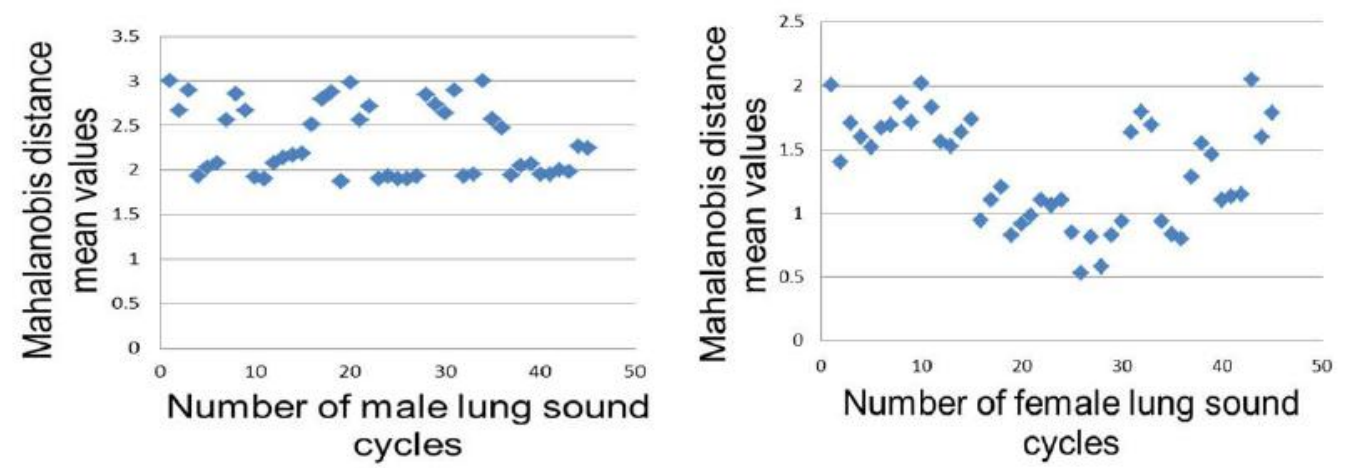

Figure 7: Mahalanobis distance mean values of each normal lung sound in healthy males and females when compared with standard normal lung sound.
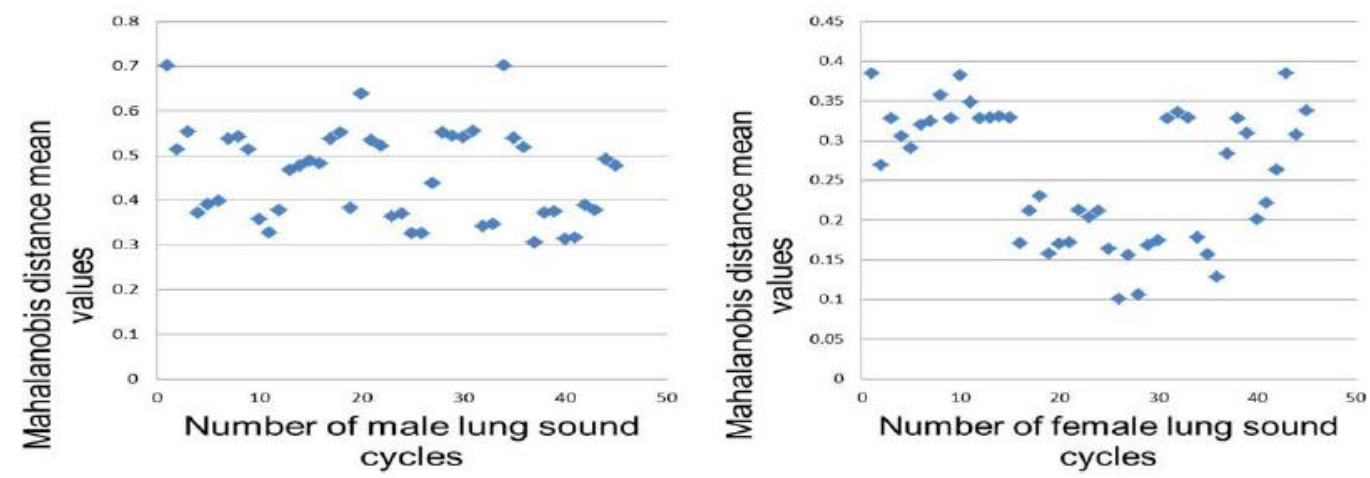

Figure 8: Mahalanobis distance mean values of each normal lung sound in healthy males and females when compared with standard abnormal lung sound.

It was observed that the values obtained for males are higher than for females and that the values are in a specific range. It was also observed that the values obtained for normal lung sounds when compared with standard normal lung sound are much closer to 1 than the values obtained for normal lung sounds when compared with standard abnormal lung sound, indicating that the normal lung sounds are much similar to standard normal lung sound. The plotted graphs were used to form ranges as shown in Table 2.

Table 2 Ranges formed using Mahalanobis distance mean values obtained for normal lung sounds.

\begin{tabular}{ccccc}
\hline & \multicolumn{2}{c}{ Male } & \multicolumn{2}{c}{ Female } \\
\hline & $\begin{array}{c}\text { Standard normal } \\
\text { lung sound }\end{array}$ & $\begin{array}{c}\text { Standard abnormal } \\
\text { lung sound }\end{array}$ & $\begin{array}{c}\text { Standard normal } \\
\text { lung sound }\end{array}$ & $\begin{array}{c}\text { Standard abnormal } \\
\text { lung sound }\end{array}$ \\
\hline Upper boundary & 3 & 0.59 & 1.9 & 0.35 \\
\hline Lower boundary & 1.8 & 0.3 & 0.7 & 0.13 \\
\hline
\end{tabular}

The Table 2 explains for an example that if a lung sound of a healthy male compared with standard normal lung sound, the Mahalanobis distance mean value should be in the range of 1.8 to 3 .

The abnormal lung sounds which were compared with standard normal lung sound and standard abnormal lung sound are shown in Figure 9 and Figure 10 respectively. 

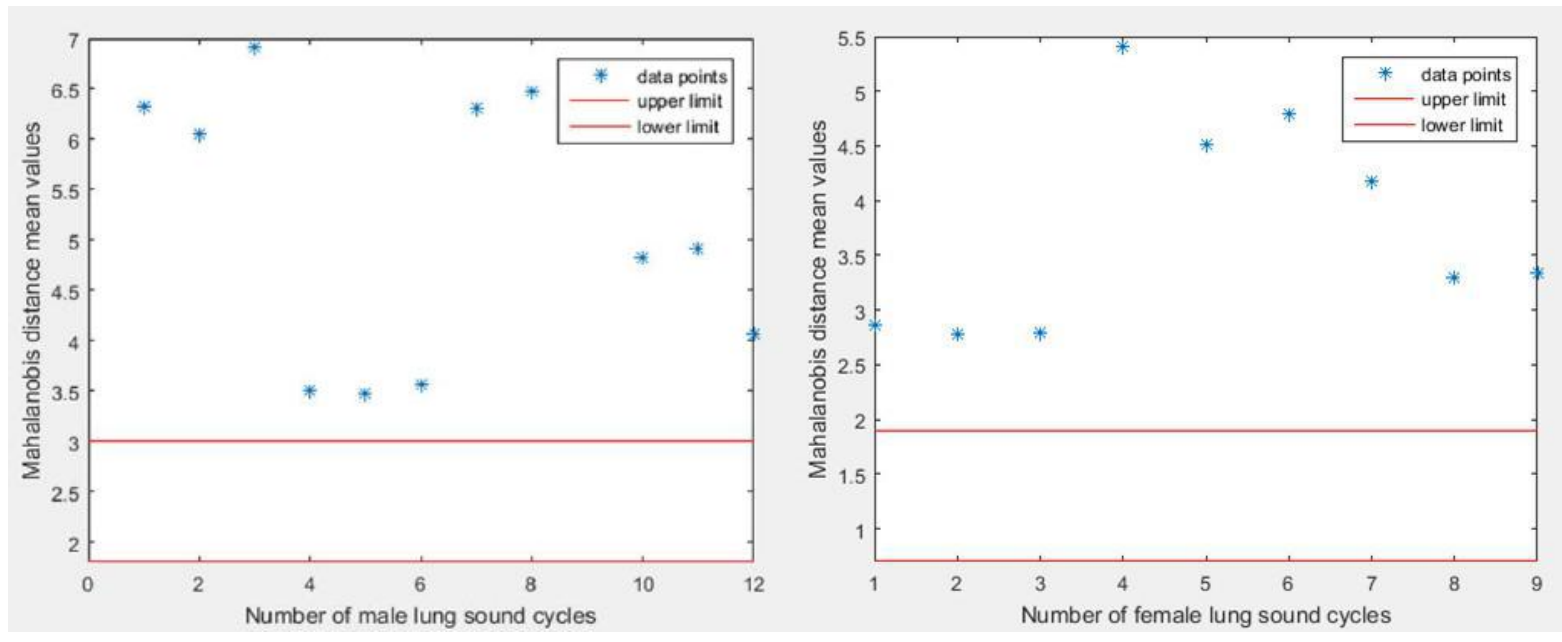

Figure 9: The data points indicate Mahalanobis distance mean values of abnormal lung sounds in males and females with respiratory disorders when compared with standard normal lung sound. Data points have deviated from the formed range which is shown by upper and lower limit.
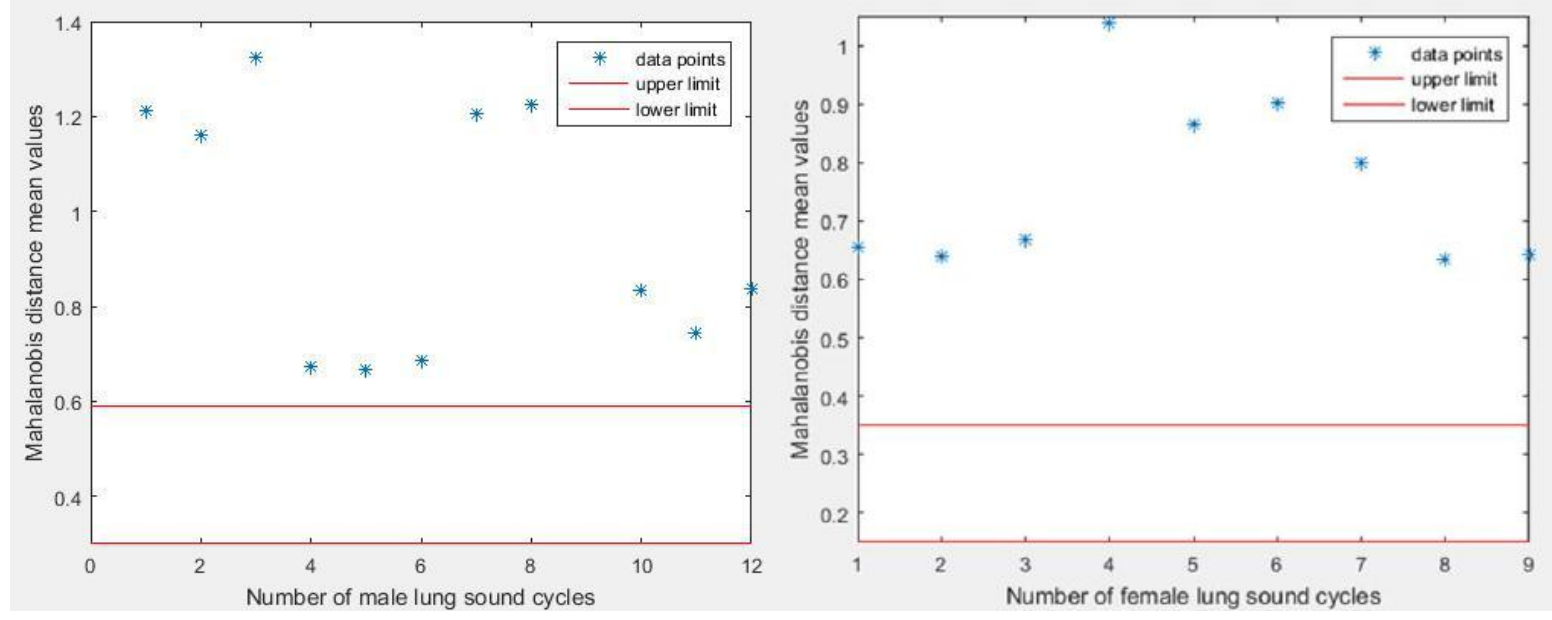

Figure 10: Data points indicates Mahalanobis distance mean values of abnormal lung sounds in males and females with respiratory disorders when compared with standard abnormal lung sound. Data points have deviated from the formed range which is shown by upper and lower limit.

It was observed that the Mahalanobis distance mean values obtained for abnormal lung sounds have deviated from the formed ranges when compared with standard normal lung sound and with standard abnormal lung sound. It was also observed that the values obtained for abnormal lung sounds when compared with standard abnormal lung sound are much closer to 1, indicating that the abnormal lung sounds are much similar to standard abnormal lung sound.

\section{Conclusion}

In this paper, a methodology has been implemented to compare normal and abnormal lung sounds and thereby to detect whether a person's lung sound is normal. It was seen that by using the Mahalanobis distance method wheeze sounds and sounds due to low lung volume can be detected as abnormal lung sounds when compared with the ranges formed using normal lung sounds and therefore with reference to the results obtained, there is feasibility in distinguishing between normal and abnormal lung sounds of individuals using Mahalanobis distance method. This study can be further carried out to the other age groups with a higher sample size as well. 
Isuri Liyanage, Githendri Siriwardhana, Anjana Abeyrathne, Asela Pallewela, Kanchana Wijesinghe / Determination Of Lung Sound As Normal Or Abnormal, Using A Statistical Technique

Also, this study can be further developed by using subjects with vast variety of respiratory disorders and this research can be conducted for all other positions of the lung.

\section{Acknowledgment}

The authors would like to thank Dr. Geethal Perera, Dr. K.L.N.S.K. De Alwis and Dr. N.D. Gajanayake for providing intellectual resources.

\section{Authors' Contribution}

I.H.H. Liyanage (liyanageharindi@gmail.com), P.K.G.Y. Siriwardhana (githendrisiriwardhana@yahoo.com) and W.H.A.U. Abeyrathne (anjanaushanthaabeyrathne@gmail.com) were with the General Sir John Kotelawala Defense University, Ratmalana, Sri Lanka (contributed equally)

Mr. Kanchana Wijesinghe was with the General Sir John Kotelawala Defense University, Ratmalana, Sri Lanka (wlpkanchana@gmail.com) (Supervisor)

Mr. Asela Lakmal Pallewela was with the Sri Jayawardhanapura General Hospital, Sri Lanka (lakmalasela88@gmail.com) (Supervisor)

\section{References}

Bouzakine, T., Carey, R., and Taranhike, G., 2005, Distinguishing between asthma and pneumonia through automated lung sound analysis. IEEE 31st Annual Northeast Bioengineering Conference, USA, April, pp. 241243.

Chang, G. C., and Lai, Y. F., 2010, Performance evaluation and enhancement of lung recognition system in two real noisy environments. Computer Methods and Programs in Biomedicine, 97,141-150.

Chowdhury, S. K., and Majumder, A. K., 1982, Frequency analysis of adventitious lung sounds. Journal of Biomedical Engineering,4,pp.305-312.

Dorantes-Mendez, G., et al., 2008, Crackles detection using a time-variant autoregressive model. 30yj Annual International Conference of the IEEE Engineering in Medicine and Biology Society, Canada, August, pp.18941897.

Guler, I., Polat, H., and Ergun, U., 2005, Combining Neural Network and Genetic Algorithm for Prediction of Lung Sounds. Journal of Medicine Systems, 29, pp.217-231.

Hadjileontiadis, L., and Panas, S., 1997, Adaptive Reduction of Heart Sounds from Lung Sounds Using FourthOrder Statistics. IEEE Transactions on Biomedical Engineering, July, 44(7), pp. 642-648.

Kanfaswamy, A., et al., 2004, Neural classification of lung sounds using wavelet coefficients. Comuters in Biology and Medicine, 34(6), 523-537.

Mahalanobis, P. C., 1936, On the generalised distance in statistics. Proceedings of the National Institute of Science of India, 12, pp. 49-55.

Matsunaga, S., et al., 2009, Classification between normal and abnormal respiratory sounds based on maximum likelihood approach. IEEE International Conference on Acoustics, Speech and Signal Processing. Taiwan, April, pp. 517-520.

McLachlan. G. J., 1999, Mahalanobis Distance. Resonance. 4(6), pp.20-26.

Potdar, R. M., et al., 2015, Implementation of Adaptive Algorithm for PCG Signal Denoising. International Journal of Innovative Research In Electrical, Electronics, Instrumentation And Control Engineering, 3(4), pp. 33-42.

Sathesh, K., and Muniraj, N. J. R., 2012, Separation of Heart Sounds from Lung Sounds Using LMS Adaptive Equalizer Implementation in Cadence. International Journal of Mechanic Systems Engineering (IJMSE), 2(1), 48-52. 
Sovijarvi, A. R. A., et al., 2000, Characteristics of breath sounds and adventitious respiratory sounds. European Respiratory Review, 10, pp. 591-596.

Vannuccini, L, et al., 2000, Capturing and preprocessing of respiratory sounds. European Respiratory Review, 10, pp. 616-620.

Zolnoori, M., et al., 2012, Fuzzy Rule-Based Expert System for Assessment Severity of Asthma. Journal of Medical Systems, 36, pp. 1707-1717. 